\section{Involucrados dentro de lo posible: Conciliación trabajo-paternidad de padres primerizos chilenos $^{*}$}

Involved as far as possible: Work-family conciliation of new Chilean fathers

\section{Yanko Pavicevic ${ }^{\star}$ \\ Florencia Herrera $^{* * *}$}

\section{Resumen}

En este artículo analizamos cómo padres primerizos chilenos intentan compatibilizar sus responsabilidades laborales con las paternales.

Esta investigación es parte del proyecto Fondecyt 1150554 "Convertirse en padre hoy: Experiencias masculinas de transición a la paternidad en Chile".

** Ayudante de investigación en la Escuela de Sociología, Universidad Diego Portales. Ejército Libertador \#333, Santiago, Chile. Correo electrónico: yanko.pavicevic@mail.udp.cl

** Docente en la Escuela de Sociología, Universidad Diego Portales. Ejército Libertador \#333, Santiago, Chile. Correo electrónico: florencia.herrera@udp.cl
Se enmarca en un estudio longitudinal que contempla el seguimiento del proceso de transición a la paternidad de 32 hombres mediante entrevistas. Para los padres es difícil conciliar el rol de proveedor con el de cuidador de sus hijos, por lo que desarrollan estrategias para estar presentes en sus vidas. Tienen intenciones de realizar cambios en sus trabajos, pero les resulta difícil concretarlos. Creemos que si se quiere avanzar hacia sociedades más igualitarias, los padres deben ser considerados cuidadores primarios de sus hijos y la crianza debe dejar de ser vista como una tarea esencialmente femenina. Las políticas de conciliación familia/trabajo deben avanzar en esta dirección.

Palabras clave: Padres, papel de los padres, investigación empírica, conciliación familia y trabajo, permiso postnatal masculino.

\begin{abstract}
This article analyzes how first-time Chilean fathers try to reconcile their paternal and work responsibilities. A longitudinal study that includes the follow up of 32 men in their transition to fatherhood was carried out applying interviews. It is difficult for fathers to reconcile the role of providers with that of care-takers of their children, so they develop strategies to be present in their lives. They have intentions to make changes in their jobs, but have trouble accomplishing them. We believe that if we want to build more egalitarian societies, fathers must be considered primary care-takers of their children and childcare should no longer be seen as an essentially feminine task. Work/family conciliation policies must move in this direction.
\end{abstract}


Key words: Fathers, parental role, empirical research, work and family balance, paternity leave.

\section{Introducción}

El presente artículo trata sobre la compatibilización que padres primerizos chilenos intentan realizar entre sus responsabilidades laborales y parentales. Se enmarca en un estudio cualitativo longitudinal en curso que busca comprender cómo viven los hombres chilenos la transición a la paternidad. Para dar cuenta de la percepción que tienen los padres sobre las exigencias paternales en lo laboral y familiar, analizamos entrevistas realizadas a 32 hombres en tres momentos diferentes (antes de la llegada del hijo/a; a los pocos meses de llegada del hijo/a; al año de llegada del hijo/a). Con el artículo buscamos aportarnuevas perspectivas al estudio de la paternidad, situándonos en Latinoamérica e incorporando paternidades no normativas como la homosexual, adoptiva y medicamente asistida. Lo hemos presentado en congresos internacionales como el VI Coloquio Internacional de Estudios sobre Hombres y Masculinidades y el XXXIV Congreso Internacional de la Asociación de Estudios Latinoamericanos, donde recibimos valiosos aportes.

La investigación sobre fertilidad, reproducción y crianza se ha centrado en la experiencia de las mujeres y en la mayoría de los casos, la experiencia de los hombres es pasada por alto (Herrera 2013; Dolan y Coe 2011; McCreight 2004; Throsby y Gill 2004). Es así como se le ha dado bastante énfasis académico y popular a la adaptación de las mujeres a la revolución de género, marcada por su entrada al mundo del trabajo, pero se le ha prestado mucha menos atención al efecto de esta revolución en los hombres, quienes han tenido que compatibilizar su rol de proveedor con el de ser padres involucrados (Lupica 2016; McGill 2014).

Existe una presión social hacia los padres para que participen activamente en la crianza de sus hijos, por parte de sus parejas, los profesionales de salud y la sociedad en general (Lupica 2015; Valdés 2009). No obstante, su círculo social, la sociedad y en muchos casos ellos mismos, siguen considerando que su principal responsabilidad es proveer a sus familias (Herrera y Pavicevic 2016). Los padres actuales conviven en la constante tensión entre proveer y cuidar (Collier 2018).

\subsection{Políticas de compatibilización trabajo y familia}

Las políticas de compatibilización de familia y trabajo suelen estar dirigidas a las mujeres. Incluso cuando están planteadas en términos -de género- neutros, en el mercado laboral nacional e internacional se asume que los hombres serán los sostenedores de sus hijos y no sus cuidadores principales (Bailey 2015; Madrid 2017; Smithson y Stokoe 2005). Se elaboran bajo el supuesto de que las tareas de cuidado y crianza de los hijos son esencialmente femeninas (Miller 2011). Por ejemplo en Chile, el derecho a sala cuna, a ausentarse del trabajo por enfermedad del hijo menor de un año y el permiso postnatal están dirigidos a las madres (Aguayo y Sadler 2011).

La política de permiso postnatal fue reformulada el año 2011 en Chile ${ }^{1}$, otorgándole a los hombres

Para más detalles se puede revisar la ley en el sitio web de la Biblioteca del Congreso Nacional (http://www.bcn.cl/leyfacil/ recurso/postnatal). 
la posibilidad de utilizar parte de la licencia postnatal de las madres de sus hijos, si ellas así lo deseaban. Esta posibilidad se sumó al permiso pagado de 5 días tras la llegada de un hijo, con el que ya contaban. Es un pequeño avance en el camino hacia la corresponsabilidad en los cuidados, ya que la licencia de paternidad es una herramienta indispensable para fomentar la participación de los hombres en el cuidado de sus hijos e hijas, reportándole una mejor salud mental y física a padres e hijos (Lupica 2016; OECD 2016). Especialmente en un contexto regional donde "las políticas de género apenas empiezan a tomar en cuenta la posibilidad de avanzar hacia la equidad incorporando a los hombres" (De Keijzer 2011: 20) en las tareas domésticas y de cuidado.

Sin embargo, de acuerdo a la legislación actual en Chile, el acceso de los hombres a un permiso postnatal extendido está sujeto a la voluntad de sus parejas, quienes deciden si les transfieren parte de su licencia a partir de la séptima semana. En realidad, es poco probable que las madres hagan uso de esta opción -y los padres la acepten- mientras están en pleno período de lactancia materna exclusiva (que abarca los primeros 6 meses de vida del hijo, al igual que el permiso parental), ya que se complicaría la alimentación de sus hijos (Lupica 2015). De hecho, entre el 2011 y el 2014 solo un $0,26 \%$ de los padres utilizaron esta posibilidad (Superintendencia de Seguridad Social 2015). Esta es una cifra normal en relación a otros países de la región y el resto del mundo, donde en general los padres utilizan muy poco de la parte transferible de las licencias parentales (Lupica 2015). Es más, en muchos países de la Organización para la Cooperación y el Desarrollo Económicos (OCDE) -entre los que está Chilemenos de 1 por cada 5 padres utiliza el permiso parental disponible para ambos padres (OECD 2016).

Finalmente, esta modificación a la legislación no ha ampliado efectivamente el postnatal exclusivo para el padre (Aguayo et al. 2011), situándonos lejos de la paridad entre licencias maternales y paternales (Kowlessar, Fox y Wittkowski 2015). La licencia postnatal reservada exclusivamente para hombres sigue siendo muy corta en Chile, incluso en comparación con los otros países miembros de la $\mathrm{OCDE}^{2}$, que en promedio reservan 8 semanas de licencia postnatal para los padres (OECD 2016).

Las políticas de conciliación de trabajo y familia que hay en Chile reflejan que es una sociedad que no se ha desligado por completo del ideal de familia industrial, donde la mujer es la responsable del ámbito doméstico -aunque trabaje- y el hombre se desenvuelve en el mundo público, asumiendo horas de trabajo en exceso (Lupica 2015; Valdés 2007). En este escenario, es socialmente aceptado que se le dé mayor libertad laboral a las madres que a los padres trabajadores, ya que "la categoría de trabajador flexible está (...) asociada a ser una mujer y tener un hijo pequeño" (Smithson y Stokoe 2005: 157). Esto deja a los hombres en una posición desfavorecida para compatibilizar sus demandas laborales y familiares. Así lo demuestra la Encuesta Nacional Bicentenario realizada el año 2011, cuyos datos reportan que un $58 \%$ de las madres encuestadas pudieron dedicarse exclusivamente a criar a sus hijos, durante sus primeros 12 meses de vida y solo el $4 \%$ reportó que el padre de sus hijos era el cuidador principal, cuando ellas trabajaban (Universidad Católica-Adimark 2011). Además,

De la que Chile también es parte desde el año 2010. 
la Encuesta Nacional sobre Uso del Tiempo (INE 2015), mostró que los hombres dedican menos de la mitad de horas diarias al trabajo no remunerado que las mujeres. Este trabajo incluye el cuidado de otros integrantes del hogar, como los hijos.

De igual modo, la encuesta IMAGES Chile $^{3}$ muestra que un $61,7 \%$ de los hombres -con hijos- de tres grandes ciudades chilenas dedican muy poco tiempo a sus hijos por motivos de trabajo y un $61,9 \%$ considera que su rol principal en el cuidado de sus hijos es ser ayudante de sus parejas, quienes son las encargadas principales de cuidarlos. Junto a esto, un $87,5 \%$ visualiza el rol de proveedor económico como su mayor responsabilidad (Aguayo, Correa y Cristi 2011).

\section{Hombres proveedores y cuidadores}

El trabajo es un factor contextual que influye en el involucramiento de los padres con sus hijos, actuando como una barrera que los separa y afectando la energía emocional que tienen para involucrarse con ellos (Chin et al. 2011; Goldberg et al. 2002). Especialmente en Chile, donde las largas jornadas de trabajo son la norma, a diferencia de Suecia u otros países más desarrollados cuyas jornadas laborales permiten dedicar más tiempo a la familia, tanto a hombres como a mujeres (Haas y Hwang 2016).

La masculinidad tradicional o hegemónica en la edad adulta "se centra en la función de

Encuesta Internacional de Masculinidades y Equidad de Género, realizada a 1.192 hombres de 18 a 59 años residentes del Gran Santiago, Gran Valparaíso y Gran Concepción y a 426 mujeres de 18 a 59 años, residentes del Gran Santiago. El trabajo de campo se realizó entre julio y agosto del año 2009 (Aguayo et al. 2011). proveedor, responsable y autoridad" (Figueroa y Franzoni 2011: 68). La capacidad de sostener económicamente a sus familias le da a los padres un sentido de legitimidad en su rol marital y paternal, ya que demuestra que son maduros y responsables (Landale y Oropesa 2001; Olavarría 2001). Mientras que tener hijos funciona como un rito de paso a la masculinidad heterosexual adulta, al probar que son sexualmente activos, viriles y heterosexuales. Ambos son mandatos esenciales del ciclo vital normativo de los hombres (Laguna-Maqueda 2016; Olavarría 2001; 2002). Para superarlos y lograr "hacerse hombres" frente a la sociedad, deben actuar de manera racional y evitar ser débiles. El principal campo de acción de los hombres está situado fuera del hogar, en el mundo público, mientras la mujer se encarga de la esfera privada (Olavarría 2001; 2002).

Sin embargo, los nuevos códigos de la masculinidad le otorgan mayor importancia a la capacidad afectiva de los hombres, y uno de sus principales canales de expresión es la paternidad (Miller 2011). La nueva masculinidad ha sido especialmente acogida por los padres jóvenes, quienes quieren diferenciarse del modelo de paternidad tradicional ejercida por sus padres o abuelos, basado en el autoritarismo y la poca sensibilidad. Buscan que la relación con sus hijos se base en el cariño y el afecto, para ser padres próximos o protagónicos en la crianza (Lupica 2016). Les interesa estar presentes en el parto, mudar a sus hijos, jugar con ellos y hacer todas las tareas parentales posibles (Lupica 2016; Valdés y Godoy 2008; Valdés 2009).

La instauración de este nuevo modelo de masculinidad no es lineal ni uniforme y convive con elementos tradicionales de la masculinidad, heredados de generaciones anteriores, como la 
concepción de que el hombre es el encargado de proveer cuando forma una familia (Elliott 2015; Madrid 2017; Valdés y Godoy 2008). Es más, en el mundo del trabajo no se ha instaurado por completo, sino que se sigue reconstruyendo y reproduciendo la masculinidad hegemónica, reforzando también la separación del trabajo y la familia (Haas y Hwang 2016; Nascimento y Segundo 2011). Incluso por parte de los mismos trabajadores, quienes pueden llegar a percibir al balance entre el trabajo y otros aspectos de la vida, como un inhibidor de sus posibilidades de ascenso (Smithson y Stokoe 2005) ${ }^{4}$.

Por otro lado, junto con todos los beneficios - "dividendos patriarcales" que tienen los hombres por vivir en una sociedad patriarcal, en la que tienen mejores posiciones, mayor riqueza y autoridad que las mujeres (Connell 2005; Olavarría 2002), también tienen algunas desventajas o "déficits patriarcales" (Bailey 2015). Una de ellas es el impedimento que tienen para dedicar el tiempo que quisieran a sus hijos, por tener que cumplir con el rol de proveedor que se les exige, pudiendo estar muy poco con sus hijos (Aguayo y Sadler 2011; Collier 2018; Valdés 2007).

En un artículo anterior, analizamos los relatos de anticipación a la paternidad de algunos de los hombres incluidos en este estudio (Herrera y Pavicevic 2016). En ese artículo concluimos que los hombres adhieren a los ideales del padre involucrado y cercano. Muchos de ellos aspiran a una parentalidad igualitaria

Esto fue documentado en un estudio de Smithson y Stokoe (2005) realizado en Inglaterra, que contrastó los discursos oficiales sobre trabajo flexible y balance vida-trabajo de organizaciones y formuladores de políticas, con los de trabajadores y gerentes de organizaciones bancarias y de contabilidad, mediante grupos focales y entrevistas individuales. en términos de género y quieren participar activa y cotidianamente en la crianza de sus hijos. Sin embargo, son ideales difíciles de alcanzar ya que no pretenden dejar de lado su rol de proveedor. Teniendo en cuenta estos antecedentes, nos preguntamos ¿de qué manera conjugan los padres estos objetivos en apariencia contradictorios?

\section{Metodología}

Los resultados expuestos en este artículo se han obtenido de un estudio cualitativo longitudinal, que contempla el seguimiento del proceso de convertirse en padre de 33 hombres chilenos. En cada caso se realizaron tres entrevistas en profundidad, una antes de la llegada del bebé ${ }^{5}$ y dos posteriores ( 2 a 4 meses después y un año después). El carácter longitudinal de este estudio nos permite observar cómo las expectativas en torno a la conciliación de sus responsabilidades parentales y laborales van variando, en la medida que se enfrentan a la experiencia cotidiana de ser padres. En el marco del estudio también realizamos una entrevista grupal $^{6}$ y un grupo de discusión ${ }^{7}$, en los que participaron 9 padres (distintos a los que participaron en las entrevistas individuales). Por lo tanto, la muestra completa para este trabajo se compone de 42 padres, 33 de los cuales fueron entrevistados individualmente y en más de una ocasión.

Se incluyen hombres que serán o han sido padres a través de la adopción.

6 Se pretendía que fuera un grupo de discusión de padres de clase media baja y baja pero llegaron solo dos participantes, por lo que se decidió hacer una entrevista grupal.

7 Tanto la entrevista grupal como el grupo de discusión fueron realizados a hombres que ya habían sido padres (y tenían hijos menores de un año). 
Las entrevistas y el grupo de discusión realizados, cuentan con una pauta muy general y flexible de preguntas y temas a abordar con cada participante. Solemos iniciar las entrevistas invitando al entrevistado a relatar sus experiencias y percepciones en torno a la etapa de la paternidad que está viviendo. En las primeras entrevistas, los hombres cuentan cómo compatibilizan las exigencias laborales con el apoyo que sienten deben entregar a sus parejas y como creen que lo harán cuando nazca el bebé. En las segundas y terceras entrevistas hablan de sus experiencias cotidianas de conciliar el trabajo y la paternidad (entre otros temas). En todas las entrevistas les solicitamos que describan con detalle un día típico del período que están viviendo, que cuenten cuáles son las principales tareas que realizan en relación a la crianza y qué les ha resultado más difícil de la paternidad. El propósito de realizar entrevistas flexibles, sin una pauta rígida, es que tanto el entrevistador como los participantes puedan desarrollar temas inesperados y la entrevista siga la dirección que el entrevistado proponga (Deave y Johnson 2008; Herrera y Pavicevic 2016; Taylor y Bogdan 1986).

Las estrategias para acceder a los participantes fueron variadas. Repartimos panfletos en la vía pública y en consultorios de salud. En el medio virtual, publicamos la convocatoria del proyecto en Facebook y en las plataformas virtuales de la Universidad Diego Portales. Utilizamos nuestra propia red de contactos, compuesta por familiares, amigos y conocidos. Asimismo, les pedimos a los mismos participantes si nos podían contactar con potenciales entrevistados (técnica conocida como 'bola de nieve'). También contactamos a una fundación de adopción, para acceder a hombres que alcanzarían la paternidad por este método. De esta forma, nuestra muestra fue construyéndose poco a poco.
Es común en los estudios de transición a la paternidad que la muestra sea homogénea y se concentre en hombres blancos, de clase media y con estudios universitarios (Deave y Johnson 2008; Draper 2003; Ives 2014; Miller 2011). Nos propusimos desde un inicio que nuestra muestra fuera lo más heterogénea y diversa posible. Uno de los aspectos más problemáticos fue incorporar a padres de distintas clases sociales, ya que los hombres de nivel socioeconómico más bajo se mostraban menos interesados y familiarizados con la investigación académica. A pesar de esto, logramos interesar a varios explicándoles los objetivos de la investigación (también utilizamos regalos de agradecimiento e invitamos a varios a tomar un café o almorzar). Dentro de la muestra, aproximadamente un tercio no tiene estudios universitarios y puede considerarse de clase media baja o baja. Los otros dos tercios cuentan con estudios universitarios y son de clase alta, media alta o media.

La mayoría de los participantes viven en la capital del país (Santiago) pero hay 6 que viven en otras ciudades (La Serena, Talca y Concepción). Su rango etario fluctúa entre los 19 y los 56 años ${ }^{8}$, pero la mayoría (16) se concentra en la cohorte de los 26 a 36 años. Asimismo, la mayoría se convirtió en padre mediante la reproducción sexual con su pareja (30 casos, calificados como padres normativos por haber utilizado el método reproductivo más común y legitimado), aunque también se incluyeron hombres que alcanzaron la paternidad mediante la adopción (8 casos) y mediante la reproducción asistida (4 casos, 2 de los cuales son hombres autodefinidos como gay). En relación a su régimen laboral, 27 de los participantes son trabajadores dependientes,

8 de ellos se ubican en el cohorte de 15 a 25 años de edad; 7 en el cohorte de 37 a 47 años de edad y 2 en el cohorte de 48 a 58 años. 
12 independientes $^{9}$ y 2 son trabajadores informales ${ }^{10}$.

Es importante resaltar, que casi todos nuestros participantes son padres muy interesados en estar presentes en la crianza de sus hijos. De acuerdo con sus relatos, pocos de ellos son padres ausentes en algún grado. Esto da cuenta de una autoselección de la muestra, que favorece la participación de hombres que se ven a sí mismos como 'buenos padres'. En este trabajo nos centramos en los relatos de los padres y no en sus prácticas, por lo que no contrastamos con observaciones de algún tipo. Nos interesaba conocer cómo ellos relataban sus experiencias.

Todas las entrevistas fueron realizadas por los autores de este artículo. En la mayoría de los casos se mantuvo al mismo/a entrevistador/a. Esto favoreció el establecimiento de un lazo de confianza con los participantes. Realizamos 79 entrevistas (32 previas a la llegada del bebé, 27 a los pocos meses y 20 al año). El promedio de duración de las entrevistas fue un poco más de una hora. Estas se llevaron a cabo en los hogares de los participantes, en cafés o restaurantes, sus lugares de trabajo y en lugares públicos (plazas o veredas). Todas las entrevistas fueron grabadas (audio) y fueron transcritas por la misma persona ${ }^{11}$.

Al momento de participar en el estudio, ya que un participante de la entrevista grupal perdió su trabajo.

10 Esta categoría incluye a quienes son los dueños de sus lugares de trabajo.

11 La transcriptora es socióloga. Antes de comenzar a transcribir se le expusieron los objetivos y principales características de la investigación. Para que comprendiera cómo debía realizar la transcripción también se le explicó cómo sería analizada la información. Se agradece la participación de Sofía Atria en la transcripción de las entrevistas.
La información recopilada en las entrevistas y en el grupo de discusión fue analizada utilizando análisis de contenido temático, que consiste en la "descomposición o fragmentación del texto en unidades constitutivas para su posterior codificación" (Ruiz 2009: 8). En este caso, la mayoría de los códigos fueron emergentes, es decir, fueron creados mientras se leían las transcripciones de las entrevistas y no a priori. Este proceso se realizó con la ayuda del software de análisis cualitativo NVivo.

El estudio en el que se enmarca este artículo fue revisado y aprobado por el Comité de Ética de la Universidad Diego Portales. Todos los participantes firmaron un consentimiento informado donde se explicaron los propósitos del estudio y las condiciones de su participación. De esta forma, nos aseguramos de que la participación fuera informada y voluntaria. La identidad de los entrevistados fue protegida a través del uso de seudónimos y la no revelación de información que pudiera permitir su identificación. A todos los participantes se les comunicó que podían retirarse del estudio cuando estimaran conveniente, de hecho uno de ellos se abstuvo de participar en la segunda entrevista $^{12}$. Pero en general, los padres se mostraron interesados en participar y algunos incluso señalaron que les resultó beneficioso el estudio, ya que los ayudó a reflexionar sobre su paternidad.

\section{Relatos de conciliación familia/trabajo}

Los padres entrevistados se identifican con el ideal de padre cercano y afectuoso, involucrado

Ya que su hija y pareja estaban viviendo fuera del país y le era muy difícil hablar de ellas. 
en la vida de sus hijos o hijas. Buscan acercarse a un modelo de corresponsabilidad en la crianza, aunque sin dejar de lado su función principal de provisión (Herrera y Pavicevic 2016; Gaba y Salvo 2016; Lupica 2015). La mayoría de sus parejas están haciendo uso de sus licencias pre o post natales y varias no tienen planeado volver a trabajar, o al menos no a tiempo completo.

Cuando nace su hijo o hija (o al momento del enlace en los casos de adopción) los padres se toman la licencia paternal de 5 días que permite la legislación chilena. Sin embargo, varios entrevistados consideran que este permiso es una 'burla' por su corta duración. Estiman que los cinco días sólo alcanzan para hacer trámites (como la inscripción del bebé en el registro civil) y no para estar con sus hijos y pareja. Es por esto que los padres se ven obligados a ocupar sus vacaciones o tomar licencias no pagadas, con el objeto de poder estar más tiempo con sus hijos recién nacidos (Aguayo, Correa y Cristi 2011). Solo un entrevistado (de clase media) utilizó semanas del postnatal de su pareja (Martín, padre adoptivo, 37-47 años), el resto no lo considera conveniente económicamente y prefiere que sus hijos estén con las madres.

Los participantes señalan que les habría encantado quedarse más tiempo en sus hogares, después del nacimiento, -como sus parejas, quienes tienen seis meses de postnatal- para involucrarse en sus familias y establecer una relación cercana con sus hijos. Varios padres sostienen que la política de permiso postnatal debiese ser más equitativa en términos de género, entre ellos Julio, quien exige un permiso postnatal de larga duración para los padres, que sea independiente del de sus parejas:
¿Pero qué necesitamos?, que nos den el permiso, que nos den la facultad legal de poder quedarnos en nuestras casas (Julio, padre normativo ${ }^{13}, 26-36$ años, segunda entrevista).

Otros participantes, no cuestionan la duración del permiso paternal y agradecen que ahora tengan cinco días libres luego del nacimiento, ya que antes no había ningún tipo de licencia para padres. Como Alfredo, quien está conforme con que "por lo menos hay un posnatal ahora" (Alfredo, padre adoptivo, 37-47 años, primera entrevista).

En las entrevistas realizadas después de la llegada de sus bebés, los hombres relatan que se les hace difícil compatibilizar las exigencias laborales con el objetivo -autoimpuesto y deseable socialmente- de ser padres cercanos y presentes en la vida diaria de sus hijos. Los horarios de trabajo y los tiempos de traslado, les dejan pocas horas libres para estar en el hogar con sus hijos o hijas. Por ejemplo, Jerónimo trabaja en una empresa que se reestructuró y volvió muy rígida y exigente (para la segunda entrevista), lo que le significa viajar más y quedarse hasta tarde en la oficina. Todo esto coincidió con el nacimiento de su hija, obligándolo a tener que compatibilizar esta mayor exigencia laboral con la nueva exigencia familiar. El trabajo le limita la relación con su hija de pocos meses de edad:

Entonces la cercanía [con el hijo] (...) yo creo que va más por el tiempo (...) dedicarle el tiempo suficiente como para generar esa cercanía. Que hoy día con el mundo laboral como te decía no... uno está medio limitado (Jerónimo, padre normativo, 26-36 años, segunda entrevista).

Ernesto, quien para la segunda entrevista trabajaba de manera dependiente y en jornada

\footnotetext{
Que fue padre producto de una relación sexual con su pareja.
} 
completa, señala que los extensos horarios de trabajo lo obligan a él y a su pareja a estar fuera de casa todo el día. Para explicar su situación, Ernesto afirma que pierde la oportunidad de estar con su hija. El trabajo es una obligación desagradable que lo hace llegar cansado a su casa y le impide compartir con su hija ${ }^{14}$ :

Es que el trabajo que tiene [su pareja] no es muy agradable entonces, es como de oficina, administrativo, con horario de 8 a 6 entonces es mucho rato afuera, o sea yo lo vivo de estar afuera todo ese rato y puta no es muy agradable, te perdiste todo el día con la niña. Y llegai al final del día y estai raja [cansado] (Ernesto, padre normativo, 26-36 años, segunda entrevista).

Antes del nacimiento de sus hijos, los hombres ya sienten que sus obligaciones laborales no les permiten estar todo lo presentes que quisieran en el embarazo, como Benjamín (paternidad normativa, 15-25 años, primera entrevista), quien cuenta que solo pudo ir a un taller de preparación parental y a algunas ecografías, ya que trabaja por turnos y le es difícil coordinar esas instancias con sus turnos de trabajo.

Para quienes trabajan de manera independiente o en lugares flexibles, les es más fácil estar presentes en la crianza. Uno de ellos es Javier, quien tiene la libertad de retirarse de su trabajo cuando su esposa lo necesite, ya que trabaja realizando proyectos de manera independiente:

O sea yo trabajo de las 9 de la mañana a las 5 de la tarde y el resto estoy todo el tiempo en la casa, eh... y hay ene veces que me llama mi señora me dice, son las tres y "oye estoy como estresadamente" y me voy no más, y me da igual.... O sea puedo hacerlo (Javier, padre adoptivo de mellizos, 37-47 años, segunda entrevista).

Su situación cambia para la tercera entrevista, ya que deja su trabajo para dedicarse a criar y trabajar desde su hogar.
Yovan trabaja de manera independiente y vive cerca de su hogar. De acuerdo con su relato, esto le da más facilidad para apoyar a su pareja durante su horario laboral: "más encima que mi departamento está acá al lado, entonces me resulta sumamente cómodo de repente si la Gabi [pareja] me pide ayuda, partir pa' allá ir no más po' " (Yovan, padre normativo, 26-36 años, segunda entrevista). Al igual que Daniel, quien dice que podrá ir a su hogar a cuidar de su hijo cuando desee, ya que su trabajo como independiente se lo permite:

\footnotetext{
Entonces lo que tenemos hablado es que cuando llegue el niño, lo más probable es que ella lo cuide $100 \%$ full y yo soy independiente entonces yo no, yo puedo ir y venir cuando quiera [a su hogar], sin..cachai (ríe)

[E] Tremenda ventaja

Sí (ríe) así que en ese sentido estamos claros, ese es como el plan me cachai (Daniel, padre adoptivo, 26-36 años, primera entrevista).
}

Sin embargo, aunque los padres con trabajos flexibles tengan mayor facilidad para acudir a los llamados de sus parejas, siguen siendo ellas las responsables del cuidado, mientras ellos se ven a sí mismos en un rol de apoyo. Además, la independencia o flexibilidad laboral tiene la desventaja de que al trabajar por metas o tener horarios flexibles, el trabajo nunca termina. Como le sucede a Jairo, quien trabaja en una universidad donde tiene un horario flexible pero debe seguir trabajando en su hogar, por lo que no puede dedicarse por completo a criar o hacer tareas domésticas cuando sale del trabajo:

Ahora en realidad yo tengo un horario bastante más flexible y... pese a que o sea yo me puedo ir a las seis de la tarde a mi casa, me puedo ir a las cinco de la tarde a mi casa, me puedo ir a las cuatro de la tarde a mi casa si quiero y uno al final siempre sigue trabajando en la casa (Jairo, padre normativo, 37-47 años, primera entrevista). 
Todos los padres dicen que intentan estar el mayor tiempo posible con sus hijos, pero sienten que sus trabajos los alejan de ellos, limitando el tiempo disponible para criar a unas cuantas horas fuera de la jornada laboral. Especialmente en los trabajos poco flexibles, como puede ser la minería o la construcción, que requieren un estilo de vida contrario a la flexibilidad (Collier, 2018). Como señalan Jerónimo y Javier el trabajo no sólo les quita tiempo si no también energía, por lo que llegan muy cansados a sus hogares, con poca energía para dedicarle a sus hijos (Chin et al. 2011; Goldberg et al. 2002). La aspiración de ser padres activos y presentes choca con el compromiso que deben demostrar en sus trabajos (Collier 2018).

En la práctica, la mayoría de los padres apoyan a sus parejas en la crianza y no comparten esta tarea de manera equitativa con ellas. Las mujeres crían a tiempo completo y los padres las relevan cuando terminan su jornada laboral, teniendo la posibilidad de elegir cuándo participar en ella (Madrid 2017). El grueso de los padres no logra cumplir con el ideal de corresponsabilidad familiar en el cuidado infantil a cabalidad (Gaba y Salvo 2016; Lupica 2015), porque su principal ocupación es el trabajo, donde muchas veces la sobrecarga es una fuerza motriz (Collier 2018). Mientras las mujeres tienen licencia postnatal o dejaron de trabajar (al menos a tiempo completo). Está la excepción de los padres gay incluidos en el estudio y un padre heterosexual normativo (Ernesto), como se verá más adelante.

La capacidad que tienen los padres estudiados de conciliar su vida familiar y laboral de manera armoniosa, varía según el tipo de trabajo que tengan y el cargo que ejerzan en él. Aquellos que trabajan de manera independiente $o$ son jefes en sus lugares de trabajo, suelen tener más libertad para manejar sus horarios laborales. Mientras quienes trabajan en lugares más rígidos y no tienen cargos de jefatura, dependen de la voluntad de sus jefes. El acceso a un determinado trabajo y puesto está condicionado por el contexto socioeconómico de cada padre y la formación que obtuvieron, siendo la mayoría de ellos profesionales. Los padres que tienen mayor flexibilidad horaria en general no cuentan con un acuerdo formal, si no que manifiestan tener un trato de palabra con sus jefes y clientes, que les permite acompañar a sus parejas e hijos a controles de salud y otras actividades.

\subsection{Mitigación del déficit patriarcal}

Los padres han desarrollado estrategias para mitigar el déficit patriarcal al que se ven enfrentados por ser los principales proveedores de sus hogares. Estas estrategias van desde adaptaciones cotidianas en sus horarios a cambios más radicales, que afectan drásticamente su vida laboral. Algunas de ellas son: tomarse más días libres cuando llega el bebé, ir a almorzar a sus hogares durante su jornada laboral, salir antes del trabajo algunos días, dedicarse completamente a sus hijos en las horas que están en la casa (antes y después del trabajo) y cambiarse o renunciar al trabajo.

La mayoría adopta estrategias moderadas, haciendo lo posible para que sus vacaciones coincidan con el postnatal masculino, y así poder estar con su pareja e hija/hijo en el primer período de la crianza. El costo es no tener días libres el resto del año. Por ejemplo, Sebastián hizo coincidir los cinco días de postnatal con tres semanas de vacaciones cuando nació su hija, permitiéndole estar un mes cuidando de ella: 
Sí, de hecho fue tan planeado [el parto] que yo... bueno (...) se diagnosticó la fecha, lo... yo planee mis vacaciones, mi semana, mis cinco días parentales con mis vacaciones, o sea todo el primer mes, y ahí estuve con la guagua así que... la última de enero, las primeras tres de febrero (Sebastián, padre normativo, 26-36 años, segunda entrevista).

Lo mismo hizo Lucas, quién trabaja en elárea de la minería con contratos cortos y no tomó trabajos después del nacimiento de su hijo, estando un mes sin trabajar (Lucas, padre normativo, 26-36 años, segunda entrevista). Otra estrategia que tienen los padres, para maximizar su presencia en el hogar es ir a almorzar a medio día (quienes viven cerca de sus trabajos). Esto les permite estar con sus hijos y apoyar a sus esposas en las tareas cotidianas. Julio es uno de ellos, ya que desde que nació su hija planea ir a almorzar todos los días a su casa:

Increíble la necesidad de contacto yo ahora voy a ir todas las semanas, todos los días a almorzar a la casa

[E] Ah qué bueno que puedas hacer eso

Si antes no lo hacía tanto, yo vivo acá en cómo te decía cerca del barrio universitario y iba almorzar, a veces almorzaba en un restaurant por acá pero no, voy a ver la guagua (Julio, padre normativo, 26-36 años, segunda entrevista).

También Ernesto, al momento de la segunda entrevista señala que va a almorzar a su casa. Argumenta que intenta apoyar lo que más puede a su pareja en las tareas domésticas y de cuidado, dentro de lo que le permite su jornada laboral:

\footnotetext{
Trabajo cerca entonces puedo venir a la hora de almuerzo, estar en la casa, apoyar con el tema de la comida, apoyar con el tema del cambio de pañal, tenerla un ratito no sé po' mientras la Sol [pareja] va al baño, cachai.. O en la mañana como, como trabajo cerca me voy muy pegado a la hora y tengo a la Carola [hija] y la Sol se va, entonces ha sido bien, la posibilidad, que el trabajo esté cerca me ha posibilitado hacer hartas cosas (Ernesto, padre normativo, 26-36 años, segunda entrevista).
}

Varios participantes afirman que antes de salir al trabajo y al volver, se encargan de sus hijos e hijas, lo que significa cambiar pañales, levantarlos, bañarlos, darles de comer y acostarlos. De esta forma, sienten que compensan -en parte- sus horas de ausencia. Esta ausencia les pesa por dos razones. La primera es que sienten que el no estar presentes afecta el vínculo que pueden desarrollar con sus bebés. La segunda es que los padres están muy conscientes de lo agotadas que están sus parejas en este período y eso les genera culpa, por lo que sienten que es su responsabilidad alivianarles la carga.

Javier, cuando sus hijos llevan un año en su familia, relata que ha reservado los miércoles en la mañana para quedarse en casa. Esto cumple el doble objetivo de estar con sus hijos y darle un espacio de descanso a su pareja. Sebastián se siente culpable por tener menor "carga de crianza" que su pareja y por lo tanto dedica todo su tiempo libre a su familia, dejando de lado su vida social y cancelando proyectos externos a sus trabajos formales.

Todos los padres afirman que intentan mantenerse conectados y entregarle apoyo emocional a sus parejas cuando no están físicamente presentes para ayudarlas, llamándolas por teléfono para saber cómo están ellas y sus hijos. Es lo que Chin et al. (2011) llaman comportamiento compensatorio. Esteban da un buen ejemplo de esto:

\footnotetext{
Eh, volver al trabajo fue (...) complejo en la relación de que, de... del tema del apego cachai como que cuesta un poco... eh, salir (...) pero igual tenís que, igual estar ahí entonces al final como hay mayor comunicación ahora me manda fotos cuando despierta, como que estamos conectados en ese sentido (Esteban, padre normativo, 26-36 años, segunda entrevista).
} 
Adolfo sostiene que se mantiene informado del estado de su hija a través de la comunicación por celular con su esposa: "(...) y de ahí me vengo [al trabajo] y... siempre me mantiene informado [su esposa], me manda fotos de ella [su hija], cómo está... eh... o qué está haciendo" (Adolfo, padre normativo, 26-36 años, segunda entrevista).

No son pocos los padres que en conjunto con sus parejas, han tomado decisiones más drásticas y han cambiado de trabajo o residencia. Como Jerónimo, quien se cambió de trabajo cuando su mujer volvió a trabajar, a uno en el que tiene horarios más estables y no tiene que viajar fuera de la ciudad. Además, se cambiaron de casa para estar más cerca de sus trabajos y de la abuela de su hija, quién los ayuda a cuidarla. Yovan comenzó a trabajar como independiente después de que nació su hija, ya que no le acomodaba estar encerrado en una oficina y quería tener mayor libertad para estar con su hija y pareja. Ahora que tiene su propia oficina puede salir cuando quiera, lo que le ha permitido asistir a todos los controles de salud de su hija:

Cachai, que en general tampoco son muchos, pero por lo menos son dos veces en la semana que las controlan a ambas, cachai, entonces... eh... yo tenía que elegir un poco [cuando era un trabajador dependiente], entonces asistía a las de la Jose [hija], para acompañar a la Gabriela [pareja] o no, entonces como que eso estaba cojo pero ahora que estoy acá yo [en su oficina independiente] va a ser más fácil (Yovan, padre normativo, 26-36 años, segunda entrevista).

Asimismo, hay padres que van incluso más allá y dejan de trabajar de manera formal o reducen su jornada laboral considerablemente. Ernesto es uno de ellos, quien no solo se retiró de su trabajo sino se fue a vivir a un pequeño balneario costero, para dedicarse por completo a criar junto con su pareja. También Alberto (padre gay por reproducción asistida, 48-58 años), quien redujo su jornada laboral a la mitad para poder estar con su hija en las tardes y Octavio, quien renunció a algunas responsabilidades en su trabajo y comenzó a trabajar media jornada (dos días a la semana), para hacerse cargo de sus hijos:

\footnotetext{
Yo antes que nacieran los niños tenía una responsabilidad sobre una región geográfica de cuatro países, estaba tres semanas fuera de Chile, si vos me decís un régimen de tres días a la semana pero yo trabajaba en un horario complejísimo. [Pareja] estuvo un año viviendo [fuera] por su trabajo, yo viajaba una vez al mes y él viajaba para acá 10 días, estábamos así...nacieron los chicos y nuestra vida cambió por completo o sea yo renuncié a toda responsabilidad fuera de Chile, me quedé solo con mis responsabilidades de acá, en un régimen en donde martes y viernes trabajo medio día para hacerme cargo (Octavio, padre gay por reproducción asistida, 48-58 años, primera entrevista).
}

En definitiva, independiente de la estrategia que tengan para lograrlo, todos los padres intentan estar lo más presentes que pueden en la crianza de sus hijos, aunque sea de manera virtual. La mayoría son padres proveedores que ajustan sus horarios laborales para tener mayor participación en la crianza de sus hijos y acompañar a sus parejas en esta tarea. En este sentido, son padres próximos pero no protagónicos en la crianza de sus hijos (Lupica 2016). Con la excepción de los 2 padres gay incluidos en el estudio (Alberto y Octavio) y un padre normativo (Ernesto), quienes se dedican casi exclusivamente a criar.

\subsection{Intenciones concretas y difusas}

Al igual que en el estudio de Stanley-Stevens y Seward $(2007)^{15}$, donde los participantes querían

15 Estudio realizado en Texas, Estados Unidos donde se aplicó un cuestionario a 25 futuros padres y se entrevistó a 14 de ellos. El trabajo de campo fue realizado el año 2000. 
involucrarse más con sus hijos que sus propios padres pero no pretendían reducir su jornada laboral, a muchos padres de nuestro estudio les gustaría quedarse en sus hogares cuidando de sus hijos pero no cuentan con un plan concreto para lograrlo, develándose como "un ideal difícil de alcanzar" (Herrera y Pavicevic 2016: 121).

Por ejemplo Moisés, en el grupo de discusión contó que tenía todo listo para retirarse de su trabajo por 6 meses y dedicarse a criar a su hija, cuando su esposa volviera a trabajar, pero no se atrevió a hacerlo:

\begin{abstract}
Pero sabes qué, yo creo que uno tiene como claro así como principios teóricos que uno quiere cumplir y otros (...) prácticos que no puede cumplir pero que en alguna medida pasa por decisiones propias, me explico, yo tengo todo este rollo de la igualdad en los roles parentales porque mis papás la tuvieron (...) y en algún minuto me propuse renunciar y estar 6 meses sin trabajar. $Y$ lo conversamos [con su esposa], incluso lo hablé con mi jefa y me arrepentí. Y no me atreví y fue pura cobardía porque yo (...) gano un poco más que mi esposa pero la diferencia es mínima. Yo (...) además tenía unos ahorros (...) con los que pude haber vivido perfectamente 6 meses, yo creo que no habría tenido problemas en reinsertarme cuando yo hubiese querido pero no me atreví y yo creo que no me atreví por, como que a lo mejor usé de excusa a mi hija para amenazar e irme del trabajo pero nunca tuve la intención seria. Entonces yo creo que hay un paso que uno no da (...), yo estoy seguro que si yo le dijera a mi jefa "no esa reunión no puede ser a las 4 porque yo a las 5 tengo que estar en mi casa porque tengo una hija" no me diría nada pero no me atrevo. Entonces yo descanso en que mi esposa va a estar a esa hora en mi casa (Moisés, padre normativo, 26-36 años, grupo de discusión).
\end{abstract}

En el relato de Moisés se evidencia la posición complicada y a la vez cómoda de los hombres en el ámbito laboral y familiar, ya que les cuesta hacer cambios en sus jornadas laborales para cumplir con sus tareas paternales adecuadamente, pero cuentan con que sus esposas harán estos cambios por ellos, aunque tengan que posponer sus carreras. Por otra parte, Iñaki dijo estar dispuesto a estar más tiempo en su hogar, pero no sabía cuando lo haría:

\begin{abstract}
Y eso creo que ella, yo quiero que vuelva a trabajar, no me gusta el modelo del papá que trabaja y la mamá que está en la casa. Creo que en muchos sentidos no es mi ideal. Este, inclusive si eso involucra que yo tenga que estar tiempo en la casa, estoy dispuesto a eso. Así que... yo pretendo que eso sea así, no sé si pase el segundo semestre o ya será el próximo año o más adelante, no tengo idea (Iñaki, padre normativo, 37-47 años, segunda entrevista).
\end{abstract}

Generalmente son las parejas de los padres quienes reducen su jornada laboral o abandonan sus trabajos para cuidar de sus hijos, liberándolos parcialmente de esta tarea. Aunque hay casos de padres que sí llevaron a cabo sus intenciones de dedicar más tiempo a criar, priorizando a la familia por sobre el trabajo. Como Yovan y Jerónimo, quienes -como se mencionó en la sección anterior- se cambiaron a trabajos menos demandantes en cuanto a tiempo o con horarios más flexibles. También están los casos de Ernesto, Alberto y Octavio. El primero se fue a vivir a un balneario costero y trabaja de manera informal desde su hogar, elaborando productos naturales y el segundo se tomó un descanso de más de un mes cuando nació su hija, y volvió a trabajar media jornada:

$Y$ he logrado fijate una cosa que me ha gustado mucho, de repente me doy cuenta que ha logrado pasar un año y tanto de tener una jornada de trabajo parcial, entonces me sigo viniendo a veces tres y media a más tardar un cuarto pa' las cuatro (Alberto, padre gay por reproducción asistida, 48-58 años, tercera entrevista).

Mientras que Octavio renunció a responsabilidades importantes en su trabajo y pidió trabajar mediajornada dos días a la semana. Estos tres últimos casos son particulares, ya que como se mencionó en la sección anterior 
son los únicos padres de nuestro estudio que crían casi a tiempo completo. Además, Alberto es un padre gay que no cuenta con el apoyo de la madre -subrogante- de su hija, aunque sí tiene el apoyo de una niñera y su pareja (con quien vive $)^{16}$. Para ellos, el éxito económico y reconocimiento social por el desempeño en sus carreras dejó de ser lo primordial en sus vidas -si es que alguna vez lo había sido- y comenzaron a serlo sus hijos (Figueroa y Franzoni 2011).

Con todo, los regímenes laborales flexibles suelen otorgársele a las madres más que a los padres, por lo que es entendible que sea difícil para ellos lograr trabajar menos horas. Vivimos en un país y un mundo generizado que clasifica a los roles reproductivos y de cuidado como fundamentalmente femeninos y a los roles productivos como principalmente masculinos (Barker y Greene 2011). Además, poder hacer un cambio efectivo en sus vidas laborales no depende solo de la voluntad de los padres, sino también de la oferta de trabajos de medio tiempo o más flexibles que haya en el mercado laboral. Cuando se le realizó la segunda entrevista a Ignacio (padre adoptivo, 26-36 años), se encontraba buscando un nuevo trabajo, ya que desde que llegó su hija no soporta hacer los turnos (de varios días) que le exige su trabajo actual ligado a la minería. Las posibilidades de encontrar este nuevo trabajo no dependen sólo de su determinación, sino que también se hayan condicionadas por factores externos ajenos a su control. Como se ha evidenciado en esta sección, la pretensión manifestada por algunos padres de dedicarse a cuidar a sus

Mientras Octavio también cuenta con el apoyo de su pareja del mismo sexo para criar, quien es el segundo padre de sus hijos y también redujo su jornada laboral. Además, ambos tienen la posibilidad de pedir apoyo a la madre de sus hijos, con quien hicieron un acuerdo de co-parentalidad. hijos no siempre tiene un correlato en la práctica (Lupica 2016; Valdés y Godoy 2008), ya sea por factores personales o externos. Esto refleja como "los discursos cambian más rápido que las prácticas" (Madrid 2017: 12).

\section{Conclusiones}

La capacidad que tienen los padres de mitigar el déficit patriarcal y compatibilizar sus trabajos con la vida familiar es variable, según las condiciones laborales de cada padre -régimen dependiente o independiente, disposición de los jefes, flexibilidad horaria- y de otros factores como la cercanía de sus hogares a sus lugares de trabajo. De todas formas, los padres no cuentan con un marco normativo que les permita compatibilizar sus trabajos con la crianza, por sobre los cinco días de licencia post natal masculina obligatoria. Las políticas estatales y del mercado laboral dirigidas a la conciliación del trabajo y la vida familiar, no proveen condiciones suficientes para que los padres dejen de ser cuidadores secundarios de sus hijos y puedan superar los patrones parentales normativos (Miller 2011). En otras palabras, aunque se ha avanzado falta mucho aún para superar el viejo modelo del hombre proveedor y la mujer dueña de casa, tan presente en Latinoamérica (Lupica 2016).

En Chile, no hay una presión política ni social que impulse a los empleadores a reducir las jornadas de trabajo de sus empleados hombres que son padres, más bien, la cultura laboral penaliza a los trabajadores que sacrifican sus compromisos laborales para cuidar a sus hijos (Lupica 2015). La expectativa cultural normativa sigue siendo que los hombres sean los principales o únicos proveedores de sus hogares y tengan limitadas responsabilidades de cuidado, especialmente 
en los primeros meses de vida de sus hijos (Haas y Hwang 2016). Para esto, las mujeres deben hacerse cargo de la crianza, dejando de lado sus trabajos.

Por otra parte, aunque los padres manifiesten la intención de seguir el patrón del 'nuevo padre', construido sobre la base de una mayor conexión, comunicación y afecto con sus hijos, esto en muy pocos casos contribuye al desmantelamiento efectivo del patrón parental normativo, más bien lo reconfigura (Madrid 2017). Pueden identificarse con el ideal del 'nuevo padre', pero una vez que nacen sus hijos en general siguen siendo los principales proveedores económicos de sus hogares y las mujeres las principales cuidadoras (Olavarría 2004; Valdés 2007). La crianza sigue siendo desigual en términos de género (Madrid 2017). Sin embargo, la forma en que cada padre estudiado ejerce su paternidad sí tiene un efecto en el significado de masculinidad compartido socialmente, contribuyendo a construirlo y redefinirlo desde un paradigma moderno, tradicional o una mezcla de ambos (Guida 2011).

Los hombres que participan en este estudio perciben que su principal tarea es proveer para sus familias, en especial cuando nacen sus hijos. Saben que serán evaluados por su entorno social según cuán buenos proveedores sean. Esto le impide a la mayoría tomar decisiones que puedan resentir su trabajo, por lo que adoptan estrategias moderadas para intentar estar más presentes en la vida de sus hijos y ayudar a sus parejas. Van a almorzar a sus hogares durante sus días laborales o se van a vivir más cerca del trabajo. Teniendo que deslizarse constantemente en el binario proveedor/cuidador (Collier 2018). Solo algunos padres (gay y normativos) se atreven a seguir estrategias más radicales, como cambiarse de trabajo o dejar de trabajar (de manera formal), ya que implica un riesgo económico. Llama la atención que los padres gay incluidos en este trabajo sigan estas estrategias más radicales, lo que da cuenta de la importancia que le otorgan a la paternidad, tras lo difícil que fue alcanzarla.

Este estudio se concentra en la primera etapa de la paternidad, por lo tanto se debe tomar en cuenta que en la mayoría de los casos las madres que trabajaban estaban con pre y post natal. Futuras investigaciones podrían indagar cómo se reordenan las tareas y responsabilidades ligadas a la crianza, cuando los hijos crecen y las mujeres vuelven a trabajar. ¿Cambia de alguna manera la posición del padre? Otra línea que valdría la pena estudiar, es la experiencia de los hombres que dejan sus trabajos para dedicarse a tiempo completo a la crianza ¿Sienten que son castigados socialmente?

Se ha escrito bastante sobre cómo la inserción de la mujer en el mundo laboral no ha ido de la mano de una mayor participación del hombre en el mundo doméstico (Olavarría 2004; Valdés 2009). Los datos nos muestran que la dedicación de los padres a los cuidados y crianza de sus hijos es muchísimo más baja que la de las madres (Aguayo et al. 2011; Universidad CatólicaAdimark 2011). Sin duda falta llevar a cabo más estudios para comprender mejor la posición en la que se encuentran los nuevos padres. Pero la información que presentamos en este artículo es un indicador de que los padres quieren estar más presentes en las vidas de sus hijos y están dispuestos a hacer cambios. Sin embargo, sienten que el trabajo es el principal obstáculo para alcanzar este objetivo. Al mismo tiempo, siguen considerando que su responsabilidad primordial es proveer para sus familias. 
Si se quiere avanzar hacia sociedades más igualitarias, la reproducción y la crianza tienen que dejar de ser vistas como tareas esencialmente femeninas. Los padres deben ser considerados cuidadores primarios de sus hijos e hijas junto con las madres. Las políticas de conciliación familia y trabajo deben avanzar en esta dirección, considerando a los hombres y promoviendo la paternidad compartida, para generar un cambio en la cultura laboral imperante.

\section{Agradecimientos}

Agradecemos el apoyo financiero del Fondo Nacional de Desarrollo Científico y Tecnológico de Chile (FONDECYT Regular $N^{\circ}$ 1150554) y el apoyo institucional de la Universidad Diego Portales. También queremos agradecer a los hombres que han confiado en nosotros y tan generosamente han compartido sus experiencias.

\section{Bibliografía}

Aguayo, F. y Sadler, M 2011. "El papel de los hombres en la equidad de género:¿qué masculinidades estamos construyendo en las políticas públicas en Chile?". Masculinidades y Políticas Públicas: Involucrando Hombres en la Equidad de Género. Aguayo, F. y Sadler, M. (Eds.). Santiago: Universidad de Chile. 105-126.

Aguayo, F., Correa, P. y Cristi, P. 2011. "Encuesta IMAGES Chile: Resultados de la encuesta internacional de masculinidades y equidad de género". CulturaSalud/EME. Disponible en: http://www.culturasalud.cl/2011/12/encuesta-images-chile. html(consultado en agosto de 2016).

Bailey, J. 2015. "Understanding Contemporary Fatherhood: Masculine Care and the Patriarchal Deficit." Families, Relationships and Societies 4 (1): 3-17. doi: 10.1332/204674314X14036152282 447.

Barker, G. y Greene, M. 2011. “¿Qué tienen que ver los hombres con esto?: Reflexiones sobre la inclusión de los hombres y las masculinidades en las políticas públicas para promover la equidad de género". Masculinidades y Políticas Públicas: Involucrando Hombres en la Equidad de Género. Aguayo, F. y Sadler, M. (Eds.). Santiago: Universidad de Chile. 23-48.

Chin, R., Daiches, A. y Hall, P. 2011. "A Qualitative Exploration of First-Time Fathers' Experiences of Becoming a Father". Community Practitioner 84 (7): 19-23.

Collier, R. 2018. "Fatherhood, Gender and the Making of Professional Identity in Large Law Firms: Bringing Men into the Frame". International Journal of Law in Context: 1-20. doi: 10.1017/S1744552318000162.

Connell, R. W. 2005. Masculinities: Second Edition. California: University of California Press.

De Keijzer, B. 2011. "Prólogo". Masculinidades y Políticas Públicas: Involucrando Hombres en la Equidad de Género. Aguayo, F. y Sadler, M. (Eds.). Santiago: Universidad de Chile. 11-21.

Deave, T. y Johnson, D. 2008. "The Transition to Parenthood:
What Does It Mean for Fathers?". Journal of Advanced Nursing 63 (6): 626-633. doi: 10.1111/j.1365-2648.2008.04748.x.

Dolan, A. y Coe, C. 2011. "Men, masculine identities and childbirth". Sociology of Health \& IIIness 33 (7): 1019-1034. doi: 10.1111/j.1467-9566.2011.01349.x.

Draper, J. 2003. "Blurring, Moving and Broken Boundaries: Men's Encounters with the Pregnant Body". Sociology of Health and IIIness 25 (7): 743-767. doi: 10.1046/j.1467-9566.2003.00368.x.

Elliott, K. 2015. "Caring Masculinities: Theorizing an Emerging Concept". Men and Masculinities 19 (3): 240-59. doi: 10.1177/1097184X15576203.

Figueroa, J. G. y Franzoni, J. 2011. "Del hombre proveedor al hombre emocional: construyendo nuevos significados de la masculinidad entre varones mexicanos". Masculinidades y Políticas Públicas: Involucrando Hombres en la Equidad de Género. Aguayo, F. y Sadler, M. (Eds.). Santiago: Universidad de Chile. 64-81.

Gaba, M. R. e Salvo Agoglia, I. 2016. "Corresponsabilidad en el cuidado infantil y conciliación con la trayectoria laboral: Significaciones y prácticas de varones argentinos." Psicoperspectivas 15 (3): 23-33. doi: 10.5027/psicoperspectivasVol15-Issue3-fulltext-749.

Goldberg, W., Clarke-Stewart, A. Rice, J. y Dellis, E. 2002. "Emotional Energy as an Explanatory Construct for Fathers" Engagement with Their Infants". Parenting 2 (4): 379-408. doi: 10.1207/S15327922PAR0204_03.

Guida, C. 2011. "Varones, paternidades y políticas públicas en el primer gobierno progresista uruguayo". Masculinidades y Políticas Públicas: Involucrando Hombres en la Equidad de Género. Aguayo, F. y Sadler, M. (Eds.). Santiago: Universidad de Chile. 83-103.

Haas, L. y Hwang, P. 2016. “'”It's About Time! ': Company Support for Fathers' Entitlement to Reduced Work Hours in Sweden". Social Politics: International Studies in Gender, State 
and Society 23 (1): 142-167. doi: 10.1093/sp/jxv033.

Herrera, F. y Pavicevic, Y. 2016. "Anticipando la paternidad: "Ella es la que está embarazada"". Masculinities and Social Change 5 (2): 107-133. doi: 10.17583/MCS.2016.2038.

Herrera, F. 2013. "'Men Always Adopt": Infertility and Reproduction From a Male Perspective". Journal of Family Issues 34 (8): 1059-1080. doi: 10.1177/0192513X13484278.

INE 2015. Encuesta Nacional sobre Uso del Tiempo. Instituto Nacional de Estadísticas. Disponible en: https://www.ine.cl/ estadisticas/menu-sociales/enut(consultado en febrero de 2019).

Ives, J. 2014. "Men, Maternity and Moral Residue: Negotiating the Moral Demands of the Transition to First Time Fatherhood." Sociology of Health \& IIIness 36 (7): 1003-1019. doi: 10.1111/14679566.12138.

Kowlessar, O., Fox, J. y Wittkowski, A. 2015. "First-Time Fathers' Experiences of Parenting during the First Year". Journal of Reproductive and Infant Psychology 33 (1): 4-14. doi: 10.1080/02646838.2014.971404.

Laguna-Maqueda, O. 2016. "Arreglos parentales de varones gay en la Ciudad de México: de la paternidad negada a la transformación inadvertida del cuidado". Masculinities and Social Change 5 (2): 182-204. doi: 10.17583/MCS.2016.2033.

Landale, N. y Oropesa, R. S. 2001. "Father involvement in the lives of mainland Puerto Rican children: Contributions of nonresident, cohabiting and married fathers". Social Forces 79 (3): 945-968.

Lupica, C. 2015. "Corresponsabilidad de los cuidados y autonomía económica de las mujeres. Lecciones aprendidas del Permiso Postnatal Parental en Chile". CEPAL. Disponible en: http:// www.cepal.org/es/publicaciones/37878-corresponsabilidadcuidados-autonomia-economica-mujeres-lecciones-aprendidas (consultado en octubre de 2016).

Lupica, C. 2016. "Licencias de paternidad y permisos parentales en América Latina y el Caribe. Herramientas indispensables para propiciar la mayor participación de los padres en el cuidado de los hijos e hijas". Masculinities and Social Change 5 (3): 295-320. doi: 10.17583/MCS.2016.2083.

Madrid, S. 2017. "The good night kiss: fatherhood among corporate managers and the reconfiguration of hegemonic masculinity in Chile". NORMA 12 (3-4): 1-16. doi: 10.1080/18902138.2017.1362536

McCreight, B. S. 2004. "A grief ignored: narratives of pregnancy loss from a male perspective". Sociology of Health \& IIIness 26 (3): 326-350. doi: 10.1111/j.1467-9566.2004.00393.x.

McGill, B. 2014. "Navigating New Norms of Involved Fatherhood Employment, Fathering Attitudes, and Father Involvement". Journal of Family Issues 35 (8): 1089-1106. doi: 10.1177/0192513X14522247.

Miller, T. 2011. Making sense of fatherhood: Gender, caring and work. Cambridge: Cambridge University Press.

Nascimento, M. y Segundo, M. 2011. "Hombres, masculinidades y políticas públicas: aportes para la equidad de género en Brasil". Masculinidades y Políticas Públicas: Involucrando Hombres en la Equidad de Género. Aguayo, F. y Sadler, M. (Eds.). Santiago: Universidad de Chile. 50-62.

Organisation for Economic Co-operation and Development. 2016. "Parental leave: where are the fathers? Men's uptake of parental leave is rising but still low". OECD. Disponible en: http:// www.oecd.org/policy-briefs/bydate/3/(consultado en agosto de 2016).

Olavarría, J. 2001. Y todos querían ser (buenos) padres: Varones de Santiago de Chile en conflicto. Santiago: FLACSO-Chile.

Olavarría, J. 2002. "Hombres: identidades, relaciones de género y conflictos entre trabajo y familia". Trabajo y Familia: ¿Conciliación?. Seminario-taller: Estrategias de conciliación, familia y trabajo con perspectiva de género. Olavarría, J. y Céspedes, C. Santiago: SERNAM, FLACSO-Chile, CEM. 53-76.

Olavarría, J. 2004. "¿Dónde está el nuevo padre? Trabajo doméstico: de la retórica a la práctica". Familia. Valdés, X. y Valdés, T. (Eds.). Santiago: FLACSO/CEDEM. 215-250.

Ruiz, J. 2009. "Análisis sociológico del discurso: Métodos y lógicas". Forum Qualitative Social Research 10 (2): Art. 26. Disponible en: http://nbn-resolving.de/urn:nbn:de:0114fqs0902263(consultado en diciembre de 2017).

Smithson, J. y Stokoe, E. H. 2005. "Discourses of Work-Life Balance: Negotiating 'Genderblind' Terms in Organizations". Gender, Work \& Organization 12 (2): 147-168. doi: 10.1111/j.14680432.2005.00267.x.

Stanley-Stevens, L. y Seward, R. R. 2007. "First Time Expectant Fathers' Attitudes, Actions and Well-Being in Regard to Work \& Family Issues". Women's Health \& Urban Life 6 (2): 81-96.

Superintendencia de Seguridad Social. 2015. "Subsidio por Permiso Postnatal Parental 2011-2014”. SUSESO. Disponible en: http://www.suseso.cl/post-natal-parental/(consultado en junio de 2017).

Taylor, S. J. y Bogdan, R. 1986. Introducción a los métodos cualitativos de investigación. México: Paidos.

Throsby, K. y Gill, R. 2004. “"It"s different for men": masculinity and IVF". Men and Masculinities, 6(4), 330- 348. doi: 10.1177/1097184X03260958.

Universidad Católica-Adimark. 2011. Encuesta nacional bicentenario. Pontifica Universidad Católica de Chile y Adimark/ GFK. Disponible en: http://encuestabicentenario.uc.cl/resultados/ (consultado en enero de 2016).

Valdés, X. y Godoy, C. G. 2008. "El lugar del padre: rupturas y herencias. Representaciones de la paternidad en grupos altos, medios y populares chilenos". Estudios Avanzados 6 (9): 79-112.

Valdés, X. 2007. "Notas sobre la metamorfosis de la familia en Chile". Futuro de las familias y desafíos para las políticas públicas. Arriagada, I. (Ed.). Santiago de Chile: CEPAL, UNIFEM, UNFPA. 41-58.

Valdés, X. 2009. "El lugar que habita el padre en Chile contemporáneo. Estudio de las representaciones sobre la paternidad en distintos grupos sociales". Polis 8 (23): 385-410. 
